\title{
XI. Kristallographische Untersuchung von Komplexverbindungen. I.
}

\author{
Von \\ Hermann Steinmetz in München.
}

(Mit 7 Figuren im Text.)

Das Ziel der chemischen Konstitutionsbestimmung ist die Erkenntnis von der Verknüpfung einer Anzahl Atome innerbalb eines chemischen Moleküls, das Ziel der kristallographischen Erforschung der Stoffe die der râumlichen Anordnung im Kristall; man kann daher letztere in gewissem Sinne als die Chemie der festen, d. h. der kristallisierten Verbindungen bezeichnen. Man hat seit Mitscherlich stillschweigend eine innige Verwandtschaft zwischen beiden Konstitutionsbestimmungen angenommen, denn sonst wären alle Schlüsse zur chemischen Konstitutionsbestimmung, die mit Verwendung der Isomorphie gemacht worden sind, sinnlos. Am weitesten in der Verwendung kristallographischer Anschauungen zur Erklärung der chemischen Natur der Komplexverbindung ging A. Werner mit dem Oktaederschema; zwar der kristallographischen Begriffswelt entnommen, hat das , Wernersche Oktaeder $₫$ freilich zunächst keine kristallographische Bedeutung, sondern ist seinem Wesen nach nur eine auschauliche Umschreibung des Begrifls der Koordinationszahl «Sechs , wie dos Quadrat die der Koordinationszahl , Vier fizierung der anorganischen Verbindungen chemisch so verschieden geartete Verbindungen wie $\mathrm{Cr}\left(\mathrm{NH}_{3}\right)_{6} \mathrm{Cl}_{3}$ und $\mathrm{Cr}\left(\mathrm{OH}_{2}\right)_{6} \mathrm{Cl}_{3}, \mathrm{SO}_{4} \mathrm{H}_{2}$ und $\mathrm{PtCl}_{4} \mathrm{H}_{2}$ einander parallel stellt, so greift er eigentlich ins Gebiet der Chemie der festen Körper über, da in diesem Falle die Eigenschaften der verglichenen chemischen Moleküle, ihre Beständigkeit, Valenzverhältnisse, Ionisation, zugunsten der zahlenmäßigen Zusammensetzung und möglichen räumlichen Anordnung vernachlässigt werden.

Die nicht strenge Unterscheidung zwischen Chemie der festen Körper und der gelösten Moleküle hat es ferner ermöglicht, daß von P. Pfeiffer ${ }^{1}$ )

1) Zeitschr. f. anorg. u. allgem. Chemie $1915,92,376 ; 1916,97,161$.

Zeitachr. f. Kristallographie. LVII. 
der Unterschied zwischen einfachen (binären) und komplexen Verbindungen verwischt wurde, indem der Koordinationszabl >Sechs * die rein geometrische Eigenschaft des flächenzentrierten kubischen Gitters, jeden beliebigen Punkt $A$ mit sechs gleichartigen und gleichartig gelagerten Punkten $B$ zu umgeben (und vice versa!), ohne weiteres gleichgesetzt wird. P. Niggli1) führt die Pfeiffersche Idee dann auf ihren wahren Wert zurück durch die Einführung der szentralen Kristallbindungenc und der selementaren Baugruppe. In den komplexen Verbindungen im weitesten Sinne werden die Bestandteile des komplexen Ions nach erfolgter Stabilisierung im Kristall allgemein als Baugruppen durch die kürzesten Abstände wieder in einem geometrisch engeren Verbande erscheinen, z. B. $\mathrm{CO}_{3}$ im Calcit2); nach dieser Begriffsbestimmung erscheint dann z. B. im Gitter des (festen) Chlornatriums jedes Atom wieder als Zentralatom, das Gitter wird also zum Spezialfall, in dem die Atome als elementare Baugruppe bei einem einzigen, also gleichen Abstand voneinander erscheinen.

Die vorliegende Abhandlung soll als erste einer Folge von Arbeiten sich mit dem Thema der Kristallographie Wernerscher Komplexverbindungen beschäftigen, das etwa so zu präzisieren wäre: Zeigen Verbindungen, in deren Molekül um ein Zentralatom sechs Reste angeordnet sind, kristallographische Eigenschaften, die das Weiterbestehen des komplexen Ions als Baugruppe im kristallisierten Zustande wahrscheinlich machen? Mit anderen Worten, hat das Wernersche Oktaeder auch für den festen Zustand eine kristallographische Bedeutung?

Es wurden zunächst Verbindungen mit der Koordinatenzabl »Sechs in Betracht gezogen; doch sind schon Versuche im Gange, die dabei gewonnenen Anschauungen auch an Viererkomplexen zu prüfen.

Hätte die Tschermaksche Regel ${ }^{3}$ ) die Bedeutung eines strengen Gesetzes, so müßte man gerade unter den Verbindungen mit der Koordinationszahl sSechs๔, zumal bei denen mit dreiwertigem Zentralatom, eine betrăchtliche Anzahl trigonal kristallisierender Substanzen finden. Das ist so wenig der Fall, daß an Verbindungen von so ausgesprochen trigonaler Formelsymmetrie wie $\mathrm{Co}\left(\mathrm{NH}_{3}\right)_{6} \mathrm{Cl}_{3}$ und $\mathrm{CoCy}_{6} \mathrm{~K}_{3}$ die Regel niemals abgeleitet worden wäre. Eine noch nicht vollendete möglichst systematisch durchgeführte Untersuchung von Kobalthexamminverbindungen wird das deutlich

1) P. Niggli, Zeitschr. für anorg. u. allgem. Chemie 1916, 94, 210.

2) Nach Johnsen, Centralbl. f. Min. usw. 1916, 385 , ist die Gruppe $\mathrm{CO}_{3}$ durch einen besonders festen Zusammenhalt ausgezeichnet, wie aus der Diskussion der Translationserscheinungen hervorgeht.

3) G. Tschermak, Tsch.s mineralog. Mitteilungen, 1903, 22, 993.

P. Niggli, diese Zeitschr. 1921, 56, 174 ff.

F. Z ambonini, Atti della $R$. Acc. usf., zitiert und erganzt in vorstehender Abhandlung P. Nigglis. 
zeigen. Daß überhaupt für Strukturen von Körpern mit den Baugruppen $A B_{3}$ die Tschermaksche Regel häufig auftritt, hat offenbar in folgendem seinen Grund: Legen wir Fig. 2 auf Seite 176 der Nigglischen Arbeit (l. c.) zugrunde, so ist jedenfalls diejenige Struktur als eine besonders stabile bevorzugt, bei der die $A, A_{1}, A_{2}, \ldots$ usw. Punkte nicht wie in der Figur in den Knotenpunkten eines rhombischen, sondern eines regelmäßigen trigonalen Gitters liegen; denn dann erhalten sämtliche Punkte der $B B^{\mathrm{I}} B^{\mathrm{II}}$ Gruppen gleichen Charakter, da sie dann mit den $A$-Punkten auf den gleichen Symmetrieachsen der betreffenden Netzebenen liegen, also der unterschiedliche Charalter von $B^{\mathrm{I}}$ und $B^{\Pi}$ gegenüber $B$ verschwunden ist. Liegen dann die $A$ - und $B$-Punkte, wie das bei einfach atomaren Komplexen wie $\mathrm{CO}_{3}, \mathrm{NO}_{3}, A s S_{3}$ vermutlich der Fall ist, in einer Ebene, so ist für die im Sinne Werners in der äußeren Sphäre liegenden - in Lösung vom Molekül abionisierenden - Atome $\mathrm{Ca}, \mathrm{Na}, \mathrm{Ag}_{3}$ eine zu der des $A-B-$ Punkts symmetrische Lage durchaus wahrscheinlich, und damit die trigonale Symmetrie des gesamten Gitters erfült. Das zahlreiche Vorkommen pseudohexagonaler Kristallstrukturen stellt Annäherungen an diesen Grenzfall dar.

Liegen dagegen die sechs um ein Zentralatom koordinierten Atome oder Atomgruppen nicht mit diesem in einer Ebene, wie es nach der Wernerschen Anschauung eben der Fall sein müßte, so sind viel mehr Möglichkeiten unsymmetrischer Anlagerung der in äußerer Sphäre stehenden Atome gegeben, und die Grundbedingung der Tschermakschen Regel könnte schon allein durch diese Tatsache in der Symmetrie des Kontinuums verschleiert werden, selbst wenn sie im Diskontinuum in der Anordnung und Parallelstellung der oktaedrischen Baugruppen vorhonden wäre. Es würde sich also in erster Linie einmal darum handeln, den Einfluß der in äußerer Sphäre stehenden Atome so weit als irgend möglich auszuschalten, d. h. Körper kristallographisch zu untersuchen, die nur aus den von sechs Resten umgebenen Zentralatomen aufgebaut sind. Dabei müßten die koordinierten Reste im günstigsten Falle aus Atomen bestehen oder die Radikale möglichst klein, chemisch gesprochen möglichst leicht sein, also aus wenigen Atơmen mit kleinem Atomgewicht bestehen. Derartige Körper wären also unter den Hexahalogeniden von Molybdän oder Wolfram zu suchen, die sich aber bisher noch nicht in genügend ausgebildetem Zustande kristallisieren ließen. Die nichtionisierenden Komplexverbindungen vom Typus des Kobalttriammintrinitrits haben zwar keine Atome in äußerer Sphäre, dagegen zwei verschiedene Radikale in der inneren, und sind infolge dieser Eigenschaft ungeeignet, grundlegende Anschauungen gewinnen zu lassen. So bleiben nur Doppelverbindungen übrig, zusammengesetzt aus einem elektropositiven und einem elektronegativen Wernerschen Oktaederkomplex,

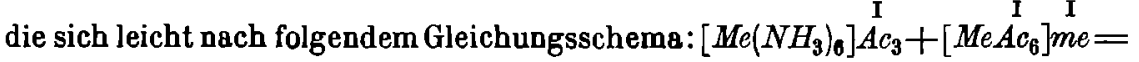


$\left[\mathrm{Me}\left(\mathrm{NH}_{3}\right)_{6}\right]\left[\mathrm{MeAc}_{6}\right]+3 \mathrm{Acme}$ darstellen lassen $(\mathrm{Me}=3 \mathrm{wertiges}$ Metall, $m e=1$ wertiges Metall, $A c=1$ wertige Säuregruppe) und die, obwohl seit langem bekannt, noch niemals kristallographisch untersucht wurden.

Solche Körper stellen wieder bināre Verbindungen dar, in denen an Stelle der Atome, z. B. des Chlors im Chlorkalium, der Rest [MeAc$\left.c_{6}\right]$, an Stelle des Kaliums der Rest $\left[\mathrm{Me}\left(\mathrm{NH}_{3}\right)_{6}\right]$ steht. Beide haben die dreifache elektrische Ladung wie ein Atom $\mathrm{Cl}$ oder $K$ infolge der Dreiwertigkeit des Zentralatoms. Nach den von Kossel vertretenen Anschauungen ist die den Zusammenhang einer solchen Verbindung darstellende s Valenz * nichts anderes als das durch den Elektronenaustausch bedingte elektrostatische Kraftfeld, das in der älteren Schreibweise durch Punkte oder Striche dargestellt wurde, ohne daß aber damit eine bestimmte Lokalisierung der Valenzkräfte angedeutet werden dürfte. Da nun die für die Kristallstruktur bestimmenden Krăfte ebenfalls elektrostatischer Natur sind(Fajans, Grim ${ }^{1}$ )), so müßten sie unabhängig von der Anzahl der das elektrostatische Feld verursachenden Elektronen sein, und Körper des genannten Typus wohl vergleichbar mit den einfachen binären Verbindungen sein. Es wird daher die Annahme eines analogen Gitters für die Schwerpunkte der diese Verbindungen bildenden positiven und negativen Komplexe zulässig sein, und wenn wir den einzelnen Komplexen eine oktaedrische Anordnung der Radikale $\mathrm{NH}_{3}$ und $\mathrm{Ac}$ um die Zentralatome $\stackrel{\mathrm{M} e}{\mathrm{Me}}$ vorlāufig zuerkennen wollen, werden noch bestimmte Vorstellungen über die Lage dieser Wernerschen Oktaeder abzuleiten sein.

Denkt man sich in einem kubischen Gitter von der Art des Chlorkaliums die $K$-Atome von den Ammino-Oktaedern, die $C l$-Atome von den AcidoOktaedern ersetzt, und zwar so, daß die Achsen der zwei Scharen von Oktaedern den Hexaederkanten des angenommenen Gitters parallel laufen, alle Oktaeder also einander parallel stehen, so folgte aus dieser Anordnung eine kubische Gesamtsymmetrie. Es wäre diese eine a priori durchaus mögliche Struktur für die in Frage stebenden Doppelkomplexe. Tatsächlich aber zeigen die Verbindungen $\left[\mathrm{Co}\left(\mathrm{NH}_{3}\right)_{6}\right]\left[\mathrm{CoCy}_{6}\right]$ mit ihren Isomorphen, wo $\mathrm{Cr}$, $\mathrm{Fe}, \mathrm{Mn}$ an Stelle von $\mathrm{Co}$ eintreten kann, sowie die entsprechenden Oxalate trigonale Kristallsymmetrie. Das würde bei parallel gestellten Oktaedern bedingen, da $B$ die Achsen der einzelnen Baugruppen-Oktaeder nicht mehr mit den Symmetrieebenen der trigonalen Gesamtstruktur zusammenfallen würden; das ist nach den aus der Tschermakschen Regel abgeleiteten Vorstellungen nicht sehr wahrscheinlich, der ja gerade ein möglichstes Zusammenfallen des Symmetriecharakters der Baugruppen mit dem der Gesamtstruktur zugrunde liegt. Nimmt man dagegen an - immer noch unter der Yoraussetzung eines kubischen Schwerpunktgitters nach Art des

1) Noch unveroffentlicht. 
Chlorkaliums - daß ein Ammino-Oktaeder in bezug auf ein Acido-Oktaeder in Zwillingsstellung nach einer Oktaederläche steht, so muß die Gesamtstruktur infolge der Verteilung der koordinierten Reste trigonale Symmetrie annehmen, trotz des kubischen Charakters des Zentralatomgitters! Diese Anordnung ist in Fig. 1 dargestellt. Die Ammino- und Acido-Oktaeder sind durch etwas verschiedene Größe unterschiedlich veranschaulicht. Oktaeder $A$ steht zu $B$ in $\mathrm{Z}$ willingsstellung in bezug auf die horizontale Oktaederebene, der Ort von $A$ entspreche z. B. einem Chlor-, der von $B$ einem Natriumatom im Chlornatriumgitter. Alle übrigen gleichartigen Baugruppen sind diesen beiden Stellungen parallel. Dadurch, daß die Oktaederflächen nicht kubische Symmetrieebenen sind, hier aber eine davon als Zwillingsebene zur Symmetrieebene zwischen zwei benachbarten Oktaedern gemacht wird, erhält nur die auf dieser bevorzugten $O$-Ebene normale Raumdiagonale die

Fig. 1.

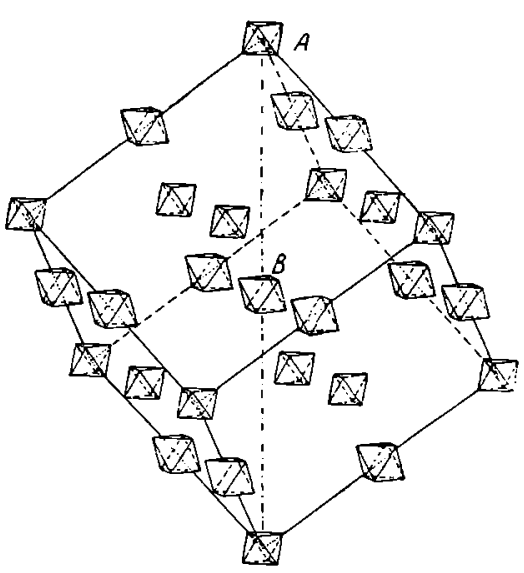
Natur einer dreizähligen Achse und unterscheidet sich dadurch von den drei übrigen untereinander gleichwertigen Raumdiagonalen des dem kubischen Schwerpunktsgitter zugrunde liegenden Einheitswürfels. Sie ist in der Fig. 1 strichpunktiert.

In dieser Ausbildung läge der Sonderfall eines $90^{\circ}$-Rhomboeders vor, mit einer im übrigen vollkommen trigonalen Natur. Tatsächlich zeigen nun die beobachteten Rhomboeder der Doppelkomplexe nicht $\alpha$-Winkel von $90^{\circ}$, sondern etwa 112 . Entweder hat demnach in der Richtung der trigonalen $c$-Achse im Vergleich mit der des $90^{\circ}$-Rhomboeders eine starke Kontraktion stattgefunden, oder es ist das (angenähert) $112^{\circ}$-Rhomboeder nicht als $R=\{100\}$, sondern als $-\frac{1}{2} R=\{110\}$ aufzufassen mit einem ibm entsprechenden $R$ von etwa $95_{2}^{\circ} \alpha$-Winkel bei sonst identischer Struktur. Die Kontraktion wäre dann nur gering, aber auch wie im ersten Falle senkrecht zu der einen Schar von abwechselnd mit lauter positiv und lauter negativ geladenen Komplexen besetzter Ebenen gelegen. Da nun die $112^{\circ}-$ Rhomboeder auch Spaltbarkeit zeigen, so läuft die Sache schließlich auf die Frage hinaus: muß man - wie bisher meist angenommen - ein Spaltungsrhomboeder unter allen Umstănden zum Grundrhomboeder nehmen, oder ist nicht ein dem $90^{\circ}$ Rhomboeder angenähertes der gleichen Struktur vorzuziehen? Bei der auch anderwärts wahrscheinlichen Verwandtschaft zwischen kubischen und trigonalen Gittern scheint diese Fragestellung durchaus berechtigt zu sein. 
Es ist natürlich nicht ganz korrekt und nur näherungsweise zulässig, in der bisherigen Art mit Wernerschen Oktaedern zu operieren. Es wäre das nur zulässig, wenn die sechs koordinierten Bestandteile aus Atomen ohne irgendwelche Eigensymmetrie beständen. Das Wiederauftreten trigonaler Symmetrie bei den Doppelkomplexen gemäß der Tschermakschen Regel scheint aber doch sehr für die oktaedrische Anordnung der koordinierten Reste zu sprechen, weil durch sie mehrere parallele, von verschiedenen Atomen besetzte Netzebenen mit zum mindesten trigonaler Schwerpunktsbesetzung sich zwanglos ergeben, die die trigonale Kontinuumssymmetrie wahrscheinlich machen. Je größer die koordinierten Reste sind, mit umso größerer Wahrscheinlichkeit werden Abweichungen von der Tschermakschen Regel auftreten, und in der Tat zeigen denn auch die Doppelkomplexe mit sechs koordinierten Harnstoflmolekülen keine trigonale Symmetrie, lassen aber in den wohlkristallisierten Trioxalatochromverbindungen noch deutliche Beziehungen zum hexagonalen System erkennen.

Andererseits werden sicher vorhandene kleine Deformationen der Wern erschen Oktaeder ohne Störung der trigonalen Symmetrie in das Gesamtgitter mit aufgenommen. So kann ein Ammoniakmolekül in der Hexammingruppe durch ein Wassermolekül ersetzt werden, und trotzdem erscheint das Hexacyano-aquopentamminsalz dem Hexamminkörper geometrisch streng isomorph. Nimmt man für das Verhältnis der von den Wernerschen Oktaedern eingenommenen Räume zu den dazwischen liegenden Răumen dieselbe Größenordnung wie für das Verhältnis von Elektronenkuben und deren Abständen voneinander an, und letztere beträgt nach $K$. Fajans und K. F. Herzfeld ${ }^{1}$ ) mehr als das Doppelte der Summen der halben Kantenlüngen der $\varepsilon$-Kuben, so wird die geringe Wirkung des Ersatzes eines Ammoniak- durch ein Wassermolekül durchaus verständlich.

Eine derartige Isomorphie * von Wasser und Ammoniak ist natürlich nicht schlechtweg als eine isomorphe Vertretung wie $M g$ und $F e, M n$ usw. zu betrachten, sondern lediglich als Folge der oben betrachteten räumlichen Verhältnisse, vielleicht vergleichbar mit jener isomorphen. Vertretung von Sauerstoff durch Fluor anzusehen, in Verbindungen wie $\mathrm{MoOFl}_{5} \mathrm{Zn} .6 \mathrm{H}_{2} \mathrm{O}$ und $\mathrm{MoO}_{2} \mathrm{Fl}_{4} \mathrm{Zn} .6 \mathrm{H}_{2} \mathrm{O}^{2}$ ); in denen spielt vermutlich die Platzfrages eine größere Rolle als der verwandte chemische Charakter von Fluor und Sauerstoff.

Wie schon erwähnt, verhalten sich die Hexammin-Trioxalat Doppelkomplexe z. B. $\left[\mathrm{Co}\left(\mathrm{NH}_{9}\right)_{8}\right]\left[\mathrm{Co}\left(\mathrm{C}_{2} \mathrm{O}_{4}\right)_{3}\right] .3 \mathrm{H}_{2} \mathrm{O}$ kristallographisch ganz wie die Hexacyanoverbindungen, kristallisieren sogar mit ganz ähnlichem Rhomboederwinkel, enthalten aber noch drei Moleküle Wasser, für welch

1) Zeitschrift für Physik, 1920, 309.

2) Vgl. Groth, Chem. Krystallogr. I, 464, 574, 602 u. a. 
letztere man also eine irgendwie trigonal symmetrische Einlagerung dieser drei Wassermoleküle annehmen muß. Es scheint, daß diese Wassermoleküle sich mit einer nur geringen Änderung der Gesamtstruktur entfernen lassen, doch konnte die mögliche Analogie zu dem Wassergehalt mancher Zeolithe mangels gewisser experimenteller Vorrichtungen, die erst noch geschaffen werden, noch nicht mit der wünschenswerten Schärfe nachgewiesen werden.

Kobalt- und Chromhexammin-hexanitritokobaltiat zeigen in winzigen mikroskopischen Kriställchen ausgesprochen pseudokubische Formen, ähnlich wie sie vom Kaliumoxyfluoro-Uranat beschrieben sind; die einzelnen Teile der komplizierten $Z$ willingsverwachsungen sind schwach doppeltbrechend, jedoch für exakte Bestimmung zu klein. Kobalt- und Chrombexamminhexarhodanatochromiat kristallisieren ebenfalls zu klein und unvollkommen, um eine sichere Kristallbestimmung zuzulassen; sie sind indessen nicht trigonal, möglicherweise aber pseudotrigonal oder pseudokubisch.

Auch Doppelverbindungen von nicht gleichwertigen Oktaederkomplexen können trigonale Kristallsymmetrie zeigen; es konnte bisher nur die Verbindung $\left[\mathrm{Cr}\left(\mathrm{NH}_{3}\right)_{6}\right]_{2}\left[\mathrm{PtCl}_{6}\right]_{3} .6 \mathrm{H}_{2} \mathrm{O}$ und die analoge $\mathrm{Co}$-Verbindung untersucht werden, denen sich das gleichfalls trigonal kristallisierende $\left[\mathrm{Cr} \mathrm{Harnst}_{6}\right]_{2}\left[\mathrm{PtCl}_{6}\right]_{3} \cdot 2 \mathrm{H}_{2} \mathrm{O}$ von Sell, gemessen von Lewis anschließt. $\mathrm{Ob}$ der Wassergehalt von zwei Molekülen nicht durch $3 \mathrm{H}_{2} \mathrm{O}$ zu ersetzen ist, ist bei dem großen Gewicht der übrigen Bestandteile nicht sicher zu entscheiden, aber wahrscheinlich. Diese Verbindungen wären abgesehen vom Wassergebalt den Sesquioxyden dreiwertiger Metalle zu vergleichen, in denen an Stelle der Atome je ein zweiwertiger bzw. dreiwertiger Oktaederkomplex eingetreten ist, einem Bauplan von Fig. 2 entsprechend. Da aber in diesem Fig. 2.

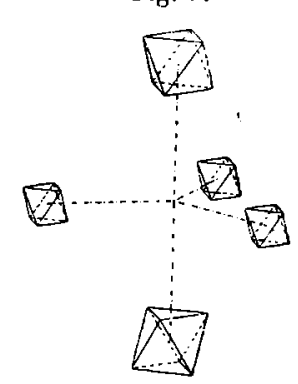
Falle schon bei der atomaren Besetzung der Gruppe $\mathrm{Me}_{2} \mathrm{O}_{3}$ eine trigyrische Raumanordnung entsteht, kann über die Stellung der $\gg$ Oktaeder $*$ nichts ausgesagt werden.

Zusammenfassend ergibt sich aus den mitgeteilten Beobachtungen: Die bei Verbindungen mit Wernerschen Oktaedern selten erfüllte Tscher maksche Regel tritt in zahlreichen Fällen wieder in Erscheinung, wenn der störende Einfluß der in äußerer Sphäre stehenden Atome oder Radikale ausgeschaltet wird. Das beweist, da $\beta$ in solchen Körpern Baugruppen mit trigonalen Symmetrieachsen vorhanden sind, eine Tatsache, die sich zwanglos aus der Annahme der Wernerschen Oktaeder ergibt. Im Verein mit den heutigen Anschauungen über Valenz und der Kenntnis der Gitter einfacher binärer Verbindungen läßt sich für die besprochenen Doppelkomplexe ein trigonal deformiertes Gitter vom Chlorkaliumtypus mit großer Wahrscheinlichkeit 
ableiten, wobei die positiven und negativen $>0$ ktaedere in Zwillingsstellung stehen.

\section{Experimenteller Teil.}

Die meisten Kristalle der im folgenden beschriebenen Körper zeichnen sich in unangenehmer Weise durch ihre Kleinheit aus, eine Erscheinung, die sich bei vielen hochmolekularen und verbăltnismăßig schwerlőslichen Kôrpern findet. Es waren häufig Flächen mit den Ausmaßen von 0,1-0,4 mm im Goniometer einzustellen; wenn die Flăchenbescheffenheit gut ist, - und das ist bei kleinen Kristallen im Durchschnitt ofters der Fall als bei großen -, so geben Flächen mit 0,22 mm mit der gewöhnlichen, etwa zweimal vergrőBernden Ableselinse $\left(>y_{\star}, F u \in B\right)$ durchaus gut einstellbare Reflexe, ohne daß man zu der Verkleinerungslinse zu greifen braucht. Die Schwierigkeit der Messung von weniger als etwa $\mathbf{0 , 5} \mathbf{m m}$ in den Durchmessern betragenden Kristallen liegt einzig in der Orientierung, d. h. im richtigen Aufsetzen, was eben Ûbungssache ist. Am vorteilhaftesten ist es, ein derartiges, kleines Kriställchen mit einer Spur Wachs an die Spitze einer abgebrochenen Nadel oder dergleichen zu setzen, und diese erst in den verschiedenen notwendigen Orientierungen auf dem Goniometertischchen mit Wachs zu befestigen; auf diese Weise wird die Berührung des Kristalls mit dem Klebemittel auf ein Mindestmaß beschränkt, und man behält leichter die Übersicht über die blank bleibenden Flächen, als wenn man den Kristall selbst jedesmal in anderer Orientierung mit Wachs anklebt. Ich wurde bei diesen oft sehr mühseligen Messungen aufs freundlichste von Herrn Dr. Franz Müller unterstützt, wofür ibm auch an dieser Stelle nochmals herzlichst gedankt sei.

\section{Doppelverbindungen aus je einem Hexammin- und einem Hexacyanokomplex.}

Es handelt sich hier um die Kombinationen der Hexacyano-, Kobalt-, Eisen-, Chrom- und Manganikomplexe mit den Hexamminkomplexen von Kobalt und Chrom. Die ersteren werden in Form der Kaliumsalze, die letzteren als Chloride oder Nitrate als Ausgangsmaterialien verwendet. Aus den jeweiligen wăsserigen Lüsungen je eines Körpers der beiden Gruppen fāllt beim Zusammengießen der beiden der entsprechende Doppelkomplex als schwerlöslicher Niederschlag aus, wãhrend Kaliumchlorid bzw. Nitrat in Lösung geht nach dem allgemeinen Schema

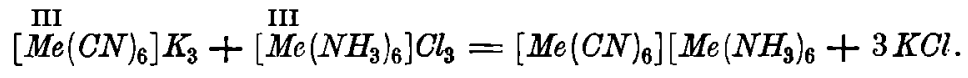

Im allgemeinen sind die Niederschläge sehr feinpulverig, aber immer kristallinisch und gut filtrierbar. Um sie in meßbarer Form zu erhalten, ist es notwendig, die Lösung der Komponenten in ein gewisses Volum Wasser $\left(\frac{1}{4}-\frac{1}{2} 1\right)$ von etwa $30-40^{\circ} \mathrm{C}$ langsam eintropfen zu lassen bis zum Beginn einer feinen Trübung; läßt man dann die Flüssigkeit recht langsam erkalten, so entstehen manchmal bis $2-3 \mathrm{~mm}$ lange und bis $0,5 \mathrm{~mm}$ dicke Kristalle. Kleine Zusätze von Salzsäure oder Ammoniak zeigen keine wesentlichen Vorteile vor rein wässerigen Lösungen. Dagegen sind die Doppelkomplexe 
in 10-20\% iger Chlorammonlösung wesentlich reichlicher als in reinem Wasser löslich, und man kann sie daher durch teilweises Eindunsten aus Chlorammonlösung umkristallisieren; wenn die Konzentration des Chlorammoniums durch fortschreitendes Eindunsten sehr groß wird, können die ausgeschiedenen Kristalle wieder in Lösung gehen. Ähnlich verhält sich auch Cyankaliumlösung.

Mit Ausnahme der Manganiverbindungen sind diese Doppelverbindungen durchaus stabile Körper, die trocken ohne Zersetzung auf $150^{\circ}$ erhitzt werden können; Umwandlungserscheinungen, die auf Polymorphie deuten, wurden dabei nicht beobachtet.

\section{Kobalthexammin-hexacyanokobaltiat $=\left[\mathrm{Co}(\mathrm{CN})_{\mathrm{B}}\right]\left[\mathrm{Co}\left(\mathrm{NH}_{\mathrm{s}}\right)_{\mathrm{A}}\right]$.}

Die Verbindung wurde zuerst von Gibbs und Genthi) später von Jörgensen ${ }^{2}$ ) dargestellt und analysiert.

$0,1749 \mathrm{~g}$ lufttrockene Substanz wurden verglüht und die gebildeten Kobaltoxyde durch mehrfaches Abrauchen mit Schwefelsäure in $\mathrm{CoSO}_{4}=0,1423 \mathrm{~g}$ übergeführt, entsprechend $30,97 \%$ Co gegen $34,33 \%$ Co berechnet; der etwas zu geringe Kobaltgehalt dürfte auf adhärierendes oder eingeschlossenes Wasser zurückzuführen sein, da bei Vorhandensein von nur einem Molekül Wasser der Kobaltgehalt auf 29,90 heruntergeht. Auch nach Jorgensen ist die Substanz wasserfrei. Die sicherste Entscheidung über den Wassergehalt würde die Elementaranalyse bringen; leider aber ist es im Augenblick infolge des niedrigen Gasdruckes nicht mōglich, im Laboratorium des mineralogischen Instituts Verbrennungsanalysen auszuführen; ein elektrischer 0 fen ist nicht vorhanden.

Kristallsystem: Trigonal. $\alpha=112^{\circ} 45_{\frac{1}{2}}^{\prime}(a: c=1: 0,4946)$.

Beobachtete Formen: $r\{100\}, a\{10 T\}$, selten $e\{111\}$.

Unter dem Mikroskop zeigen die Kristalle bäufig pseudorhombendodekaedrischen Habitus; bei vorsichtigem Kristallisieren herrscht die Prismenzone vor und zeigt etwas Längsstreifung. Fig. 3.

$$
\text { Berechnet: Beobachtet: }
$$

$$
\begin{array}{lc}
r: r=(100):(010)=- & 50^{\circ} 521^{\prime} \\
r: c=(100):(111)=29^{\circ} 44^{\prime} & \text { ca. } 30 \\
a: a=(101):(110)=60 \quad 0 & 5959
\end{array}
$$

Deutlich spaltbar nach $r$.

Optisch einachsig; das Interferenzbild zeigt beim Drehen zwischen gekreuzten Nikols keine Unregelmäßigkeit. Optisch positiv.

Farbe rötlich orange, kaum erkennbarer Dichroismus, die Schwingungen parallel der $c$-Achse (extraord. Strahl) sind reiner gelb, senkrecht dazu etwas blasser mit einer Nuance nach Braun.

Fig. 3.

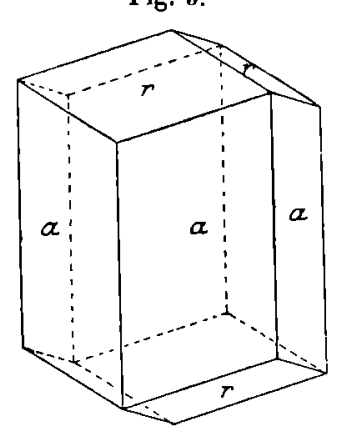

Spez. Gewicht: 1,700 bei $15^{\circ}$.

1) Researches on the Ammonia-Kobalt-Bases. Washington 1856, 48. - Journ. f. prakt. Chemie 1857, 72, 161.

2) Journ. f. prakt. Chemie 1887 [2], 35, 447. 
Kobalthexammin-hexacyanoferroat $=\left[\mathrm{Co}\left(\mathrm{NH}_{\mathrm{8}}\right)_{\mathrm{e}}\right]\left[\mathrm{Fe}(\mathrm{CN})_{\mathrm{e}}\right]$.

Zuerst dargestellt von Gibbs und Genth (l. c.); später von Braun 1).

In kaltem Wasser nahezu unlöslich, löst sich merklich in warmem, doch scheidet sich daraus die Verbindung nicht unzersetzt ab, sondern es bilden sich nur braune Häute. Ziemlich leicht löslich in warmer, etwa 20\% iger Chlorammonlüsung, beim Erkalten Ausscheidung von meßbaren Kristallen. Auch aus Lösungen in sehr verdünnter Salzsäure sind Kristalle zu gewinnen, solange nicht die Erhöhung der $\mathrm{HCl}$-Konzentration durch Eindunsten zersetzend einwirkt.

Trigonal; $\alpha=112^{\circ} 46^{\prime}(a: c=1: 0,4943)$.

Beobachtete Formen: $r\{100\}, a\{10 \bar{T}\}$. Meist nach der Prismenzone mehr oder weniger verlängerte Pseudorhombendodekaeder.

$$
\begin{aligned}
& \text { Berechnet: Beobachtet: } \\
& \begin{array}{l}
r: r=(100):(010)=- \\
r: c=(100):(111)=29^{\circ} 43^{\prime}
\end{array} \\
& a: a=(10 \mathrm{~T}):(1 \mathrm{~T} 0)=60 \quad 0 \quad 60 \quad 0
\end{aligned}
$$

Das Interferenzbild konnte wegen der Kleinheit der Kristalle nicht erhalten werden.

Optisch positiv. Farbe orangegelb, ohne erkennbaren Dichroismus.

Deutlich spaltbar nach $r$.

Kobalthexammin-hexacyanomanganiat $=\left[\mathrm{Co}\left(\mathrm{NH}_{8}\right)_{6}\right]\left[\mathrm{Mn}(\mathrm{CN})_{6}\right]$.

Die Verbindung fält $z$ war sofort beim Zusammenbringen äquimolekularer Lösungen von Kobalthexamminsalz und Manganicyanidcyankalium aus als bräunlich-fleischroter Niederschlag, zersetzt sich aber mit Wasser so rasch, daß alle weiteren Versuche mit diesem Körper unmöglich waren.

Kobalthexammin.hexacyanochromiat $=\left[\mathrm{Co}\left(\mathrm{NH}_{8}\right)_{\mathrm{B}}\right]\left[\mathrm{Cr}(\mathrm{CN})_{8}\right]$.

Zuerst dargestellt von Braun ${ }^{2}$ ), dann von Pfeiffer und Haimann ${ }^{3}$, die diesen Körper hinsichtlich seiner Isomerie mit der Chromammin-Kobaltcyanidverbindung chemisch untersuchten.

Meßbare Kristalle wurden wieder aus Chlorammonlösung erhalten.

Trigonal; $\alpha=112^{\circ} 51 \frac{1}{2}^{\prime}(a: c=1: 0,4911)$.

Beobachtete Formen: $r\{100\}, a\{10 \bar{T}\}$. Meist prismatisch nach $a$, weniger pseudorhombendodekaedrisch.

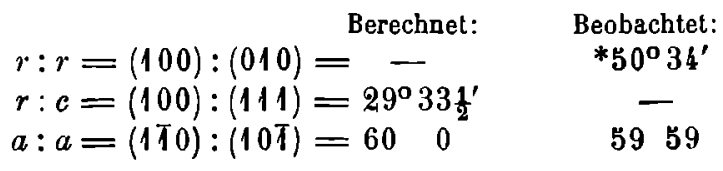

1) Annalen der Chemie 1863, 125, 182.

2) Annalen der Chemie 1906, 346, 73.

3) Journ. f. prakt. Chemie 1884 [2], 30, 30. 
Ungestörtes Interferenzbild optisch einachsiger Kristalle. Optisch positiv. Farbe orangegelb; sehr schwacher Dichroismus, $\varepsilon$ etwas heller als $\omega$. Deutlich spaltbar nach $r$.

\section{Chromhexammin-hexacyanokobaltiat $=\left[\mathrm{Cr}\left(\mathrm{NH}_{\mathrm{8}}\right)_{\mathrm{B}}\right]\left[\mathrm{Co}(\mathrm{CN})_{\mathrm{a}}\right]$.}

Zuerst dargestellt von Jörgensen ${ }^{1}$ ), dann von Pfeiffer und Hai$\operatorname{mann}($ l. c.).

Kristalle sowohl aus Chlorammoniumlösung als aus langsam abkühlender Lösung in Wasser.

Trigonal; $\alpha=112^{\circ} 46^{\prime}(a: c=1: 0,4943)$.

Beobachtete Formen: $r\{100\}, a\{10 \bar{\pi}\}$. Prismatisch nach $a$, auch Pseudorhombendodekaeder.

$$
\begin{aligned}
& \text { Berechnet: Beobachtet: } \\
& r: r=(100):(010)=-\quad * 30^{\circ} 50 \frac{1}{2}{ }^{\prime} \\
& r: c=(100):(111)=29^{\circ} 43^{\prime} \\
& a: a=(110):(10 \pi)=60 \quad 0 \quad 60 \quad 0
\end{aligned}
$$

Interferenzbild nicht beobachtet. Optisch positiv.

Farbe gelb, ohne erkennbaren Dichroismus.

Deutlich spaltbar nach $r$.

Chromhexammin-hexaoyanomanganiat $=\left[\operatorname{Cr}\left(\mathrm{NH}_{8}\right)_{\mathrm{B}}\right]\left[\operatorname{Mn}(\mathrm{CN})_{\mathrm{B}}\right]$.

Die Verbindung fällt sofort beim Zusammengießen der Lösungen der Komponenten als gelblich brauner Niederschlag aus. Im Vergleich mit dem analogen Kobalthexamminsalz ist sie etwas beständiger, zersetzt sich aber in wässeriger Lösung immer noch ziemlich rasch unter Ausscheidung brauner Manganhydroxyde. In sehr verdünnter Salzsäure sehr rasch, in Chlorammoniumlösung etwas weniger rasch als in reinem Wasser zersetzlich. Auch Zusatz von Glycerin oder Mannit zur Lösung in Chlorammonium führte nicht zu meßbaren Kristallen. Dagegen gelang es, aus warmer Cyankaliumlösung (etwa 10\% ig) sehr kleine, aber doch meBbare Kristalle zu erhalten. Die trockene Substanz wird in einigen Tagen zum Teil braun und scheidet Mangan- und Kobaltoxyde aus.

Trigonal; $\alpha=112^{\circ} 47^{\prime}(a: c=1: 0,4939)$.

Beobachtete Formen: $r\{100\}, a\{10 T\}$. Nach der Prismenzone schwach verlängerte Pseudorhombendodekaeder.

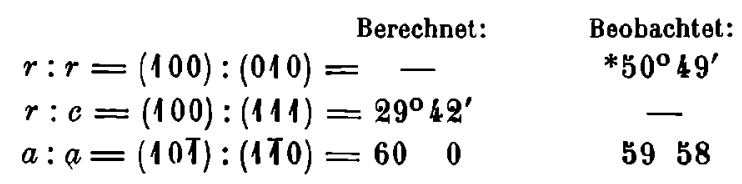

Farbe bräunlichgelb, $\varepsilon$ etwas heller als $\omega$. Optisch positiv. Deutlich spaltbar nach $r$.

1) Journ. f. prakt. Chemie 1884 [2], 30, 30. 
Chromhexammin-hexacyanoferriat $=\left[\operatorname{Cr}\left(\mathrm{NH}_{\mathrm{z}}\right)_{\mathrm{e}}\right]\left[\mathrm{Fe}(\mathrm{CN})_{\mathrm{e}}\right] \cdot$

Zuerst dargestellt von Jörgensen (l. c.). Meßbare Kristalle wurden aus Chlorammonlősung erzielt.

Trigonal; $\alpha=112^{\circ} 42^{\prime}(a: c=1: 0,4968)$.

Beobachtete Formen: $r\{100\}, a\{10 T\}$. Prismatisch nach der $c$-Achse.

$$
\begin{aligned}
& \text { Berechnet: Beobachtet: } \\
& r: r=(100):(010)=-\quad * 51^{\circ} 03^{\prime} \\
& \begin{array}{ll}
r: c=(100):(111)=29^{\circ} 50 \frac{1}{2}^{\prime} & - \\
a: a=(10 T):(1 T 0)=60 & 0
\end{array}
\end{aligned}
$$

Interferenzbild nicht beobachtet. Optisch positiv.

Farbe hellorangegelb, $\varepsilon$ etwas heller als $\omega$.

Deutlich spaltbar nach $r$.

\section{Chromhexammin-hexaejanochromiat $=\left[\operatorname{Cr}\left(\mathrm{NH}_{8}\right)_{6}\right]\left[\operatorname{Cr}(\mathrm{CN})_{\mathrm{B}}\right] \cdot$}

Zuerst dargestellt von Jörgensen (1. c.).

$0,1456 \mathrm{~g}$ Substanz ergaben nach dem Verglühen $0,0608 \mathrm{~g}^{\mathrm{Cr}_{2} \mathrm{O}_{3}=}$ 28,61\% $O r$ gegen 28,73\% $C r$ berechnet.

MeBbare Kristalle durch langsame Abkühlung der Lösung der Komponenten.

Trigonal; $\alpha=112^{\circ} 59^{\prime}(a: c=1: 0,4906)$.

$$
\begin{aligned}
& \text { Berechnet: Beobachtet: } \\
& r: r=(100):(010)=-\quad *_{50}^{\circ} 32^{\prime} \\
& r: c=(100):(111)=29^{\circ} 311^{\prime}{ }^{\prime} \\
& a: a=(10 \mathrm{~T}):(1 \mathrm{~T} 0)=60 \quad 0 \quad 6001
\end{aligned}
$$

Interferenzbild ungestört. Optisch positiv.

Farbe gelb, am hellsten von allen derartigen Körpern ohne erkennbaren Dichroismus.

Deutlich spaltbar nach $r$.

Der Kobalthexamminkomplex erteilt den beschriebenen Verbindungen einen etwas tieferen, d. h. mehr gegen Rot liegenden Farbenton als der analoge Chromhexamminkomplex; unabhāngig davon ist jedoch der Charakter der Doppelbrechung, der bei allen Verbindungen positiv ist. Alle zeigen ferner eine nicht gerade vollkommene aber deutliche Spaltbarkeit nach dem Rhomboeder, und im Verein mit der großen Winkelähnlichkeit sind diese Verbindungen wohl als isomorphe Gruppe aufzufassen. 
Doppelverbindungen aus einem Aquopentemmin- und einem Hexacyanokomplex.

Kobaltaquopentammin.hexacyanokobaltiat $=\left[\mathrm{Co}\left(\mathrm{NH}_{\mathrm{z}}\right)_{5} \mathrm{H}_{2} \mathrm{O}\right]\left[\mathrm{Co}(\mathrm{CN})_{\mathrm{e}}\right]$

Zuerst dargestellt von Gibbs und Genth (l. c.), dann Braun (l. c.), Christensen ${ }^{1}$ ) und Jörgensen (l. c.). Nach letzterem enthält die Verbindung ein Mol. Wasser, was aber nach den vollkommen analogen Eigenschaften der Kristalle wie bei den Kristallen der Hexamminreihe nicht wahrscheinlich ist. Die Entscheidung könnte wieder nur die Elementaranalyse treffen, die von keinem der Autoren ausgeführt wurde.

Der Körper ist in Wasser etwas löslicher als die entsprechende Hexamminverbindung, und man erhält immer meßbare Kristalle, wenn man etwa $\frac{1}{4} 1$ Wasser von $30^{\circ}$ mit den Lösungen von Aquopentamminchlorid und Kobaltikaliumcyanid bis zur eben beginnenden Ausscheidung versetzt und langsam abkühlen läßt.

Trigonal; $\alpha=112^{\circ} 25_{\frac{1}{2}}^{\prime}(a: c=1: 0,4991)$.

Beobachtete Formen: $r\{100\}, a\{10 T\}$, meist als Pseudorhombendodekaeder ausgebildet mit wenig verlängerter Prismenzone.

$$
\begin{array}{lc}
r: r=(100):(010)=- & \begin{array}{c}
\text { Berechnet: } \\
\text { Beobachtet: }
\end{array} \\
r: c=(100):(111)=29^{\circ} 57^{\prime} & -141^{\prime} \\
a: a=(1 \bar{T} 0):(10 \mathrm{~T})=60 \quad 0 & 600
\end{array}
$$

Normales Interferenzbild einachsiger Kristalle; prächtig granatrote Farbe, schwacher Dichroismus; die Schwingungen des ordentlichen Strahles sind etwas tiefer, mehr gegen blau, die des außerordentlichen mehr etwas gegen gelb gefärbt. Optisch positiv.

Deutliche Spaltbarkeit nach $r$.

Spez. Gewicht: 1,730 bei $15^{\circ}$.

Kobalthexammin-hexacyanoferroat $=\left[\mathrm{Co}\left(\mathrm{NH}_{8}\right)_{5} \mathrm{H}_{9} O\right]\left[\mathrm{Fe}(\mathrm{CN})_{\mathrm{G}}\right]$ -

Zuerst dargestellt von Gibbs und Genth (l. c.).

Die Verbindung kristallisiert weniger gut als das Kobaltsalz.

Trigonal; $\alpha=112^{\circ} 291^{\prime}(a: c=1: 0,4979)$.

$$
\begin{aligned}
& \text { Berechnet: Beobachtet: } \\
& r: r=(100):(010)=-\quad * 51^{\circ} 8 \frac{3^{\prime}}{4} \\
& r: c=(100):(111)=29^{\circ} 54^{\prime} \\
& a: a=(10 \mathrm{~J}):(1 \mathrm{~T} 0)=60 \quad 0 \quad 60 \quad 0
\end{aligned}
$$

Farbe bräunlich rot; $\varepsilon$ mehr nach gelb, $\omega$ mehr. nach rot gefărbt. Optisch positiv.

Deutlich spaltbar nach $r$.

1) Journ. f. prakt. Chemie 1881 [9], 23, 50. 
Kobaltaquopentammin-hexacyanochromiat $=\left[\operatorname{Co}\left(\mathrm{NH}_{\mathrm{3}}\right)_{6} \mathrm{H}_{\mathbf{2}} \mathrm{O}\right]\left[\mathrm{Cr}(\mathrm{CN})_{6}\right]$.

Zuerst dargestellt von Christensen (1. c.).

Kristallisiert wie die Kobaltverbindung gut.

Trigonal; $\alpha=112^{\circ} 423^{\prime}(a: c=1: 0,4948)$.

Beobachtete Formen: $r\{100\}, a\{10 T\}$. Vorwiegend pseudorhombendodekaedrischer Habitus.

$$
\begin{array}{lc}
r: r=(100):(010)=- & \begin{array}{c}
\text { Berechnet: } \\
\text { Beobachtet: } \\
* 50^{\circ} 53^{\prime}
\end{array} \\
r: c=(100):(111)=29^{\circ} 441^{\prime} & - \\
a: a=(10 T):(1 T 0)=60 \quad 0 & 60 \quad 0
\end{array}
$$

Normales Interferenzbild einachsiger Kristalle.

Farbe der des Kobaltiats sehr ähnlich; $\varepsilon$ etwas gelblicher als $\omega$. Optisch positiv.

Deutlich spaltbar nach $r$.

Die Ersetzung eines Moleküles Ammoniak durch ein Molekül Wasser in dem ursprünglichen Hexamminkomplex macht sich also hauptsächlich nur in der Verschiebung der Farbe von Orange nach Rot bemerkbar, während die übrigen Eigenschaften nicht prinzipiell geāndert werden.

\section{Doppelverbindungen aus je einem Hexammin- und je einem Hexanitrokomplex.}

\section{Kobalthexammin-hexanitritokobaltiat $=\left[\mathrm{Co}\left(\mathrm{NH}_{\mathrm{g}}\right)_{\mathrm{a}}\right]\left[\mathrm{Co}\left(\mathrm{NO}_{\mathrm{q}}\right)_{\mathrm{g}}\right]$.}

Diese Verbindung wurde zuerst von Jörgensen ${ }^{1}$ ) dargestellt. Sie fällt beim Versetzen von Lösungen der Kobalthexamminsalze mit Lösung von Kobaltinatriumnitrit als sehr schwer löslicher, feinpulveriger gelber Niederschlag aus, und es war nicht möglich, sie durch Variationen der Făllungsbedingungen oder Umkristallisieren aus verschiedenen Lösungen von Ammonsalzen oder Nitriten in deutlich meßbarer Form zu erhalten. Unter dem Mikroskop ist nur der pseudokubische Charakter dieser Verbindung festzustellen; man erhält sehr hăufig solche, die im Typus ganz denen von Kaliumoxyfluorouranat ${ }^{2}$ ) gleichen; oft sind die Einzelindividuen der Durchwachsungszwillinge noch länger prismatisch ausgebildet, so daß sechsstrablige Sternchen entstehen, die auch Jörgensen schon beobachtet hat; vielfach beobachtet man nur pseudooktaederische Gebilde. Es ist schwache Doppelbrechung vorhanden, aber keine genaue optische Orientierung möglich. Die Verbindung mit Chrom an Stelle von Kobalt im Hexamminkomplex verhält sich ganz ähnlich.

1) Zeitschr. f. anorg. Chemie 1894, 5, 177.

9) Groth, Chem. Kryst. I, 1906, 688, Fig. 382. 
Doppelverbindungen von je einem Hexammin- und je einem Hexarhodanatokomplex.

\section{Kobalthexammin -hexarhodanatochromiat, Trihydrat $=$ $\left[\mathrm{Co}\left(\mathrm{NH}_{8}\right)_{8}\right]\left[\mathrm{Cr}\left(\mathrm{CN}_{3}\right)_{6}\right] \cdot 3 \mathrm{H}_{2} \mathrm{O}$.}

Beim Versetzen einer Kobalthexamminsalzlösung mit nur kleinen Mengen von Chromkaliumrhodanid fält in feịnen glānzenden Schüppchen die Doppelverbindung aus. Ganz ebenso verhält sich Chrombexamminsalz.

Beide Verbindungen, Kobalt- wie der Chromkörper, sind äußerst schwer Jöslich und ließen sich weder durch verschiedene Ausfällungsbedingungen noch durch Lösen in Rhodansalzlösungen in eine kristallographisch bessere Form bringen, als sie beim langsamen Ausfällen entsteht.

Trocken erhitzt schmilzt die Chromverbindung zu einer schwärzlichgrünen Masse, die dann bei andauerndem Erhitzen ins Glühen gerät und ohne weitere Wärmezufuhr zu $\mathrm{Cr}_{2} \mathrm{O}_{3}$ verglimmt.

$0,1040 \mathrm{~g}$ Subst. ergaben $0,0262 \mathrm{~g} \mathrm{Cr}_{2} \mathrm{O}_{3}=17,25 \% \mathrm{Cr}$ gegen $17,13 \%$ theoret.

$0,3072,, \quad 0,0768,,=17,11 \%,, 17,13 \%$,

Infolge der ungünstigen Kristallisationsverhältnisse ist eine sichere Kristallbestimmung nicht möglich. Das Kobaltiat zeigt im Mikroskop sehr kleine rechtwinkelige Blättchen mit diagonaler Auslöschung; der Winkel der Begrenzungskanten ist indessen nicht ganz konstant, sondern schwankt zwischen $90-94^{\circ}$. Fig. 4. Manche der kleinen Blāttchen machen den Eindruck von Rhomboedern mit einer vorherrschend ausgebildeten Fläche; da aber über die Flächenwinkel nichts auszusagen ist, kann ebensogut eine monokline Kombination vorliegen. Das Chromiat zeigt ebenfalls diagonal auslöschende Blättchen, auch häufig Wachstumsformen; auch hier erscheint der Begrenzungswinkel nicht konstant, doch fanden sich keine rechtwinkeligen Formen. Vorwiegend ist die längere Diagonale die Fig. 4.

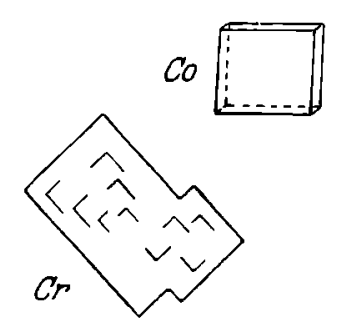
Schwingungsrichtung der rascheren Welle, es kommt aber auch der gegenteilige Fall vor, was beweist, daß die Begrenzungskanten nicht von normal gebildeten Flächen gebildet sind.

Andere Rhodanatoverbindungen mit dreiwertigen Komplexen anderer dreiwertiger Metalle darzustellen, gelang nicht. Das von Vanino ${ }^{1}$ ) dargestellte Trikaliumwismuthrhodanid gibt mit Hexamminchloriden keine Doppelkomplexe, sondern zerfällt in Lösung. Auch Zusätze von Mannit, Glyzerin, Rhodansalzen führten nicht zu greifbaren Verbindungen.

1) Zeitschr. f. anorg. Chemie 1906, 48, 215. 
Doppelverbindungen von einem Hexammin- und je einem Trioxaletokomplex.

Kobalthexammin-trioxalatokobaltiat, Trihydrat $=$

$\left[\mathrm{Co}\left(\mathrm{NH}_{\mathrm{g}}\right)_{\mathrm{B}}\right]\left[\mathrm{Co}\left(\mathrm{C}_{\mathrm{g}} \mathrm{O}_{4}\right)_{\mathrm{g}}\right] \cdot 3 \mathrm{H}_{\mathrm{g}} \mathrm{O}$.

Zuerst dargestellt von Pfeiffer und Basci ${ }^{1}$ ).

Der Körper fălit beim Zusammengießen von Lösungen von Kobalthexamminsalz und Kaliumchromioxalat sofort als feinkristalliner Niederschlag aus, läßt sich aber, wenn die Lösungen vorsichtig in Wasser von etwa $30^{\circ}$ eingetropft werden, durch langsames Ablkühlen in kleinen, aber doch meßbaren Kristăllchen erhalten.

Trigonal; $\alpha=111^{\circ} 05^{\prime}(a: c=1: 0,5528)$.

Beobachtete Formen: $c\{111\}, a\{10 T\}, r\{100\}$. Vielfach Kombination von $a$ und $c$, meist $a$ vorherrschend, aber daneben immer auch Plättchen von $c$ mit $a$ untergeordnet, daneben auch zahlreiche Kombinationen mit $r$.

$$
\begin{array}{lr}
\text { Berechnet: } & \begin{array}{r}
\text { Beobachtet: } \\
* 32^{\circ} 34^{\prime}
\end{array} \\
r: c=(100):(111)=- & 5529 \\
a: a=(10 T):(1 T 0)=60 \quad 0 & 600
\end{array}
$$

Durch sehr dünne basische Plättchen ist eben noch das dunkle Kreuz des normalen Interferenzbildes einachsiger Kristalle zu sehen, jedoch nur an so dünnen Plättchen, daß die Bestimmung des Charakters mit Gipsoder Glimmerplättchen nicht mehr möglich ist. Die Farbe der Substanz ist ein tiefes Schwarzgrün; die Einzelkristāllchen zeigen einen prachtvollen Dichroismus: $\varepsilon$ indigblau, $\omega$ turmalingrün.

Die Spaltbarkeit ist unvollkommen nach $r$ und $a$.

Beim Erhitzen und beim längeren Liegen über Schwefelsäure im Exsikkator wird die Substanz rot, ohne jedoch völlig zu einem Pulver zu verwittern. Die kleinen Prismen bleiben dabei klar, löschen zwischen gekreuzten Nikols einheitlich aus und zwar parallel der c-Achse, das Interferenzbild durch basische Kriställchen gesehen, verschwindet oder wird wenigstens undeutlich und scheint sich aus verschiedenen Anteilen mit je einer normal zur Basis stehenden Achse von zweiachsiger Substanz zusammenzusetzen. Demnach scheint sich die ursprünglich trigonale Verbindung in einen pseudotrigonalen Körper umgewandelt zu haben und zwar so, daß die āußere Form und auch ein Teil der inneren Struktur sich gesetzmäßig und im wesentlichen ähnlich erhalten haben. Diese Umwandlung ist begleitet von einem Gewichtsverlust, der aber, wie mehrfache Versuche zeigten, nicht scharf zu definieren ist, jedenfalls immer den Gewichtsverlust des theoretischen Wassergehalts überschreitet. Möglicherweise

1) Annalen der Chemie 1906, 346, 32. 
kann auch ein Stellungswechsel von Ammoniak und Wasser eintreten, Verhältnisse, die erst durch die gleichzeitige Bestimmung von Wasser und Kohlen- und Stickstoff durch Elementaranalyse geklärt werden können.

Mit diesem Salz sind noch analog die Verbindungen mit $\mathrm{Cr}$ und $\mathrm{Co}$ zusammengesetzt, die nur Prisma und Basis als Kristallbegrenzung zeigten und deren wichtigste Eigenschaften folgende Tabelle zeigt:

Farbe

Dichroismus

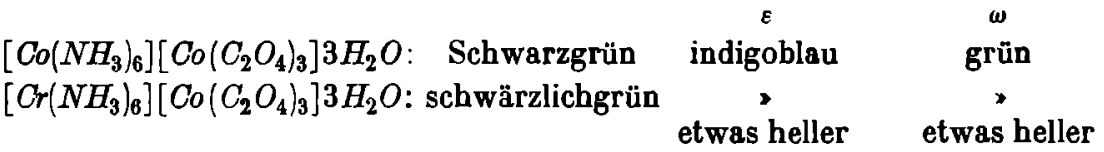

$\left[\mathrm{Co}\left(\mathrm{NH}_{3}\right)_{8}\right]\left[\mathrm{Cr}\left(\mathrm{C}_{2} \mathrm{O}_{4}\right)_{3}\right] 3 \mathrm{H}_{2} \mathrm{O}$ : violettbraun rötlich-blau grünlich-hellbraun $\left[\mathrm{Cr}\left(\mathrm{NH}_{3}\right)_{6}\right]\left[\mathrm{Cr}\left(\mathrm{C}_{2} \mathrm{O}_{4}\right)_{3}\right] 3 \mathrm{H}_{2} \mathrm{O}$ : grünlichstahlgrau, kornblumen- olivgrün bis sehr dunkel blau olivbraun, je nach Dicke.

Äußerlich sehen sich die beiden Körperpaare mit dem gleichen Metall in der Oxalatogruppe ähnlich; allen gemeinsam ist die verhältnismäßig geringe Absorption von $\varepsilon$ im Blau und für $\omega$ im Grün. Aber da beide Farben ziemlich geringen Helligkeitswert haben, erklärt sich die Undurchsichtigkeit von relativ sehr dünnen Substanzschichten. Sie zeigen alle das normale Interferenzbild optisch einachsiger Kristalle, doch ließ sich der optische Charakter daran nicht mehr bestimmen.

Doppelkomplexe aus je einem Chromhexaharnstoffund Hexacyanokomplex.

Chromhexaharnstoffchlorid wurde aus $\mathrm{CrCl}_{3} 6 \mathrm{H}_{2} \mathrm{O}$ nach P. Pfeiffer ${ }^{1}$ ) dargestellt; die Lösung des trigonal kristallisierenden Kürpers ${ }^{2}$ ) gibt mit den Lösungen von Kobalti-, Ferri- und Chromicyankalium sofort Niederschlage der Doppelverbindungen, die aber nie in meßbaren Kriställchen erhalten werden konnten. Sie zeigen im Mikroskop übereinstimmende Formen:

Fig. 5.

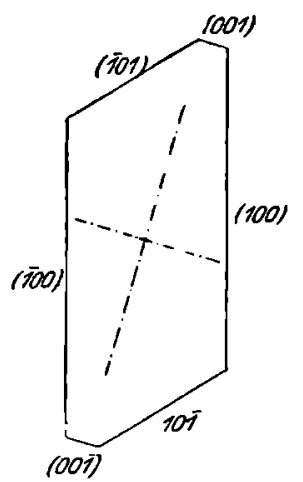

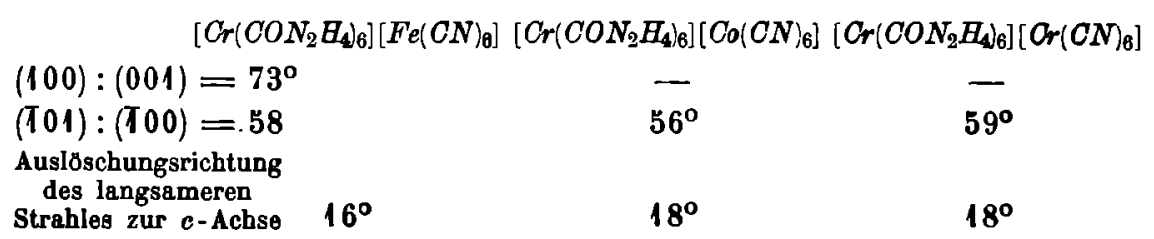

1) Ber. d. d. chem. Ges. 1903, 86, 1926.

2) Lewis, Proceed. Roy. Soc. Lond. 1889, 45, 321. Ausz. Zeitachr. f. Krist. 80, 96. Zeltachr. f. Kriatallographle. IVII. 
Schwacher Dichroismus; die raschere Schwingung ist etwas tiefer gefărbt.
Farbe: Gelbgrün
Blaßbraungrün
Blaßblaugrün.

Über etwaige pseudotrigonale Symmetrie ist außer der Annäherung der Winkel $(100):(001)$ an $60^{\circ}$ nichts auszusagen.

\section{Chromhexacarbamid-trioxalatochromiat, Trihydrat, Racemat $=$ [CrHarnst.o $]\left[\mathrm{Cr}\left(\mathrm{C}_{2} \mathrm{O}_{4}\right)_{\mathbf{3}}\right] \mathrm{BH}_{\mathbf{2}} \mathrm{O}$.}

Diese Verbindung 1) kristallisiert aus der warmen Lósung, der die Komponenten, Heraharnstoffchromchlorid und Kaliumchromioxalat enthaltenden Flüssigkeit bald nach dem Zusammengießen der äquimolekularen Anteile ziemlich langsam aus, und bildet von all den beobachteten Doppelkomplexen die gr6ßten Kristalle. Beim trockenen Erhitzen verliert die Substanz unter Trübung zuerst Wasser, schmilzt dann und gerät schließlich ins Glühen, das sich ohne Zufuhr von Wärme durch die ganze Masse fortplanzt.

$0,1482 \mathrm{~g}$ Subst. ergaben $0,0290 \mathrm{~g} \mathrm{Cr}_{2} \mathrm{O}_{3}$ entsprechend 13,39\% $\mathrm{Cr}$ gegen 13,20 ber.

$0,1830>>0,0358$, > $>13,40 \%$,

Triklin asymmetrisch; $a: b: c=0,6076: 1: 0,7079 ; \quad a=99^{\circ} 04^{\prime}$; $\beta=99^{\circ} 15^{\prime} \frac{1}{2}^{\prime} ; \gamma=91^{\circ} 46^{\prime}$.

Beobachtete Formen: $c\{001\}, \gamma\{00 T\}, b\{010\}, \beta\{0 T 0\}, m\{110\}, m^{\prime}\{10\}$, $\mu\{1 T 0\}, \mu^{\prime}\{110\}, l\{0 \overline{2} 1\}, \lambda\{02 T\}, i\{0 \overline{2} T\}, p\{1 T 1\}, \pi\{T 1 T\}, \omega\{T T 1\}, f\{1 T T\}$, $x\{131\}, \xi\{\overline{3} T 1\}$. Fig. 6 .

Der Habitus der Kristalle ist langprismatisch nach der Zone [100]; das vordere Ende ist meist gut ausgebildet, das hintere keilt in unregel-

Fig. 6.

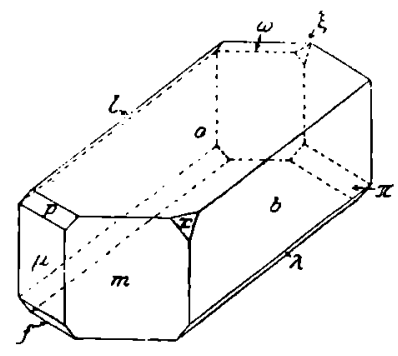
mäßige Spitzen und Wachstumsformen aus, selten sind beiderseitig ausgebildete Kristalle. Die Lănge der Kristalle beträgt meist einige Millimeter, manchmal auch Zentimeter, die Dicke überschreitet selten $1-2 \mathrm{~mm}$. Die $\mathrm{Zu}-$ gehörigkeit zur asymmetrischen Klasse beweist die häufig verschiedene Ausbildung der Prismenenden, sowie das konstant einseitige Auftreten der Flächen $i, \omega, t, x$ und $\xi$. Der pseudohexagonale Charakter ist einmal ausgeprägt durch die Zone [001], sowie durch die Kombination von Formen des Typs $\{111\}$ mit $\{021\}$, die zusammen die bekannte pseudohexagonale Pyramide bilden. $x$ und $\xi$ sind stets klein. Alle Flāchen zeigen lebhaften Glanz.

1) Da die Verbindungen mit dem Komplex $\left[\mathrm{Cr}\left(\mathrm{C}_{2} \mathrm{O}_{4}\right)_{3}\right]$ : in je einer Linksund einer Rechtsform auftreten, so ist auch wahrscheinlich der vorliegende Doppelkomplex in zwei steroisomeren Formen existent. In dem Temperaturintervall von $40^{\circ}$ bis $10^{\circ}$, in dem bisher kristallisiert wurde, findet eine Spaltung in Rechts- und Linksform nicht stalt; eine chemische Trennung wurde noch nicht ausgeführt, und es wird erst später darüber berichtet werden. Vgl. A. Werner, Ber. d. d. chem. Ges. 1912, 45, 3061 und F. M. Jaeger, Rec. Trav. Chim. Pays-Bas 1915, 38, 247. 


\begin{tabular}{|c|c|c|}
\hline \multirow[b]{2}{*}{ Berechnet: } & & \\
\hline & Beobachtet: & Beobachtet: \\
\hline$(001):(010)=-$ & $* 80^{\circ} 31^{\prime}$ & $80^{\circ} 42^{\prime}$ \\
\hline$(010):(110)=-$ & *56 20 & 5541 \\
\hline$(110):(110)=-$ & *62 30 & 6131 \\
\hline$(110):(0 T 0)=61^{\circ} 10^{\prime}$ & 6110 & - \\
\hline$(001):(110)=77 \quad 5$ & 778 & 7638 \\
\hline$(001):(110)=-$ & *86 35 & - \\
\hline$(0 \pi 0):(0 \overline{2} 1)=-$ & *38 39 & 3838 \\
\hline$(0 \pi 0):(0 \overline{2} T)=32 \quad 15$ & 3210 & - \\
\hline$(111):(001)=5032$ & 5038 & - \\
\hline$\left(1 T_{1}\right):(0 \pi 0)=7359$ & 7402 & - \\
\hline$(\pi T 1):(001)=6248$ & 6253 & 一 \\
\hline$\left(T_{T}\right):\left(0 T_{0}\right)=6636 \frac{1}{2}$ & 6636 & - \\
\hline$(1 T T):(00 T)=5451$ & $54 \quad 49$ & 5508 \\
\hline$(1 T T):(0 T 0)=6008$ & 6009 & - \\
\hline$(0 \overline{2} T):(T T 0)=57 \quad 18$ & 5720 & - \\
\hline$(0 \overline{2} 1):(1 \bar{T} 0)=62 \quad 18 \frac{1}{2}$ & 6219 & - \\
\hline$(3 T 1):(T T 0)=2626 \frac{1}{2}$ & 2617 & - \\
\hline$(131):(010)=33 \quad 0$ & 3307 & - \\
\hline
\end{tabular}

Spaltbarkeit: Vollkommen nach $c\{001\}$ und $b\{010\}$, deutlich nach $m\{110\}$.

Optische Eigenschaften: Die Ebene der optischen Achsen liegt nabezu senkrecht zur Zone [100]; auf $b$ ist das Interferenzbild der einen optischen Achse angenähert normal zu $b$; Achsenwinkel ziemlich klein, deutlich asymmetrische Dispersion. Die Farbe der Kristalle ist dunkel blaugrūn; wenn grőßere Kristalle gezüchtet sein werden, wird der Dichroismus noch genau bestimmt werden.

Die isomorphe Kobaltverbindung kristallisiert nur in sebr kleinen, aber wegen ihres hohen Flächenglanzes doch noch meßbaren Kriställchen; ihre Ausbildung ist einfacher, die gemessenen Winkel sind mit in voriger Tabelle angegeben. Sie ist grünschwarz und nur in dünnsten Splittern durchscheinend. Fig. 7.

Auf $c$ außert sich an der Chromverbindung die Ätzung auf $c\{001\}$ mit etwas erwärmter Mutterlauge in Riefen, die der Kante (001):(131) parallel laufen, während auf $\gamma\{00 T\}$ nur unregelmäßige Figuren entstehen. Diese Erscheinung war auf einer Anzahl von Kristallen ganz gleichartig, auch bezüglich der Orientierung der Winkel, so

daß von den vier möglichen Ausbildungsweisen triklin asymmetrischer Kristalle nur eine Art vorliegt.

Fig. 7.

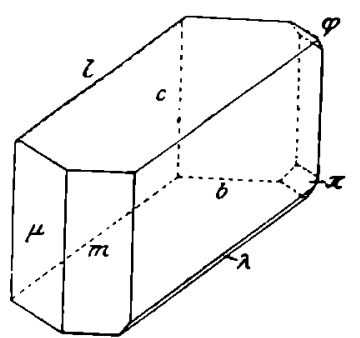


252 Hermann Steinmotz. Kristallogr. Untersuchung von Komplerverbindungen. I.

Doppelverbindungen von zwei Hexammin- mit drei Hexachlorokomplexen.

Kobalthexammin-hexachloroplatinat $=\left[\mathrm{Co}\left(\mathrm{NH}_{\mathrm{g}}\right)_{\mathrm{B}}\right]_{\mathrm{g}}\left[\mathrm{PtCl} \mathrm{Cl}_{\mathrm{B}}\right]_{\mathbf{2}} .6 \mathrm{H}_{\mathrm{g}} \mathrm{O}$.

Zuerst dargestellt und analysiert von Rogojski ${ }^{1}$; dann von Gibb und Genth (l. c.). Dargestellt wurde die Verbindung nach Jörgensen ${ }^{2}$ ) aus Natriumplatinchlorid und Kobalthexamminitrat.

Die Kristalle sind sehr klein und zeigen nur die Kombination eines hexagonalen Prismas mit der Basis. Interferenzbild optisch einachsiger Kristalle, optisch negativ.

Farbe dunkel goldgelb, ohne deutlichen Dichroismus.

Chromhexammin-hexachloroplatinat, Hexahydrat $\left.=\left[\mathrm{Cr}\left(\mathrm{NH}_{\mathrm{a}}\right)_{6}\right][\mathrm{PtCl}]_{0}\right]_{6} \mathrm{BH}_{2} \mathrm{O}$.

Zuerst dargestellt von Jörgensen ${ }^{3}$ ). Die Verbindung fällt direkt als goldgelber Niederschlag beim Versetzen einer Chrombexamminlösung mit einer solchen von Natriumplatinchlorid und läßt sich aus warmem Wasser umkristallisieren.

$$
\begin{gathered}
0,1450 \mathrm{~g} \text { ergaben } 0,0651 \mathrm{~g} \mathrm{Cr}_{2} \mathrm{O}_{3}+P t \text { beim Verglühen }=44,90 \% \\
\text { gegen } 44,97 \% \text { theor. }
\end{gathered}
$$

Die sehr kleinen Kriställchen sind sehr scharf ausgebildet, zeigen aber nur ein hexagonales Prisma mit Basis. Das Interferenzkreuz ist an den dickeren Kristallen eben zu erkennen. Optisch negativ, ohne erkennbaren Dichroismus.

1) Ann. Chim. Phys. [3], 1852, 41, 445.

2) Journ. prakt. Chemie 1887 [2], 35, 422.

3) Ebenda 1884 [2], 30, 15. 